\title{
Fully Automatic Shape Modelling Using Growing Cell Neural Networks
}

\author{
Luca Ferrarini, Hans Olofsen, Mark A. van Buchem, \\ Johan H.C. Reiber, and Faiza Admiraal-Behloul \\ LKEB - Leiden University Medical Center, The Netherlands \\ L.Ferrarini@lumc.nl \\ http: / /www. Ikeb.nl
}

\begin{abstract}
In this paper, we present a new framework for shape modelling and analysis: we suggest to look at the problem from a pattern recognition point of view, and claim that under this prospective several advantages are achieved. The modelling of a surface with a point distribution model is seen as an unsupervised clustering problem, and tackled by using growing cell structures. The adaptation of a model to new shapes is studied as a classification task, and provides a straightforward solution to the point correspondence problem in active shape modelling. The method is illustrated and tested in 3D synthetic datasets and applied to the modelling of brain ventricles in an elderly population.
\end{abstract}

\section{Introduction}

Statistical shape modelling has been increasingly used during the last decade as a basis for image segmentation, interpretation and the studying of shape changes. Many successful 2D-applications have been described in literature [1]. The model building requires the establishing of correspondence between shape surfaces over a set of training examples. Defining correspondent points on different shapes is not trivial: in some 2D applications, manual landmark definition might be possible but it becomes unpractical when 3D/4D shapes are considered. Different techniques have been developed to address this problem: some solutions are rooted in computer graphics [2], others in signal/image processing [3], [4], [5], and some have used information theory [6], [7], [8]. An important distinction has to be made between pairwise and groupwise approaches (see [6] and [8]): groupwise analysis aims to optimize an objective function over the whole dataset while creating the statistical model, while pairwise solutions generally start with a good representative of the dataset and build up a model rooted on it. In the final discussion, we shortly present advantages and drawbacks of these approaches, justifying the research on the pairwise approach described in this work.

From a pattern recognition prospective, and considering a pairwise approach, the problem can be summarized in 3 questions: How many nodes are needed? Where should they be located? How can we define correspondence between them? The question "how many nodes are needed?" is tackled as an unsupervised learning (clustering) problem where the optimal number of clusters (nodes) has to be defined. To ensure an optimal location of the points, the clustering technique needs to be topology-preserving. We 
consider the point correspondence as a classification problem where the generalization aspect of a classifier is used to match unseen cases to similar previously seen points. We investigated the use of growing artificial neural networks to tackle all three questions. Most of the growing neural networks are variations of the growing cell structure (GCS) introduced by Fritzke in [9]. The GCS principle is based on Self Organizing Maps (SOM) (Kohonen [10]), which are known to be perfectly topology-preserving: the network preserves neighborhood relations in the data by mapping neighboring inputs onto neighboring nodes in the map. Recently, Marsland et al. presented a self-organizing network that grows when required (SONGWR) [11]: SONGWR proved to be (1) more data-driven while growing, and (2) faster in learning input representation, when compared with previous models.

In this paper we describe how the SONGWR is used to create a first point distribution model from a representative instance of the training set (a single case or an average). The adaptation phase of the SONGWR algorithm is then used to deform and match the obtained network to all the instances in the training set. We investigated the robustness and effectiveness of the method in synthetic data sets. Finally, we show a successful application of the algorithm in the modelling of brain ventricles in magnetic resonance images.

\section{Method}

Given a dataset of segmented objects $T=\left\{D_{1}, \ldots, D_{n}\right\}$, we detect the clouds of points $P_{1}, \ldots, P_{n}$ by selecting the boundary points of each instance. Afterwards, the method goes through two main phases: an unsupervised clustering is used to create a first model of a representative object $P_{i}$ (chosen through visual inspection); subsequently, an adaptation phase adapts the model to the other clouds of points, keeping the point correspondence.

\subsection{Unsupervised Clustering: How Many Nodes?}

The unsupervised clustering algorithm (SONGWR) is fully described in [11]: we summarize it here, highlighting the main variations we introduced:

1. initialize a network $M$ with 2 nodes at random positions

2. do

(a) randomize the order of the points in $P_{i}$

(b) for each point $p \in P_{i}$

i. identify the two best matching nodes $s, t$ in the network $M$ :

$s=\arg \min _{n \in M}\|p-n\|, \quad t=\arg \min _{n \in M-\{s\}}\|p-n\|$

ii. set $e d g e_{a g e_{s, t}}=0$ (age of $e d g e_{s, t}$ ), or create the edge if not existing

iii. evaluate the activity of $s$ as: $\quad a=e^{-\|p-s\|}$

iv. if $a<a_{T}$ and $s_{\text {age }}>a g e_{T}$

- add a new node: $\quad r=(s+p) / 2$

- insert $e d g e s_{r, s}, e d g e_{r, t}$, and remove $e d g e_{s, t}$

v. else

- adapt $s$ position: $\quad s^{t+1}=w_{\text {winner }} * s_{\text {age }}^{t} *\left(p-s^{t}\right)$ 
- adapt nodes $i \in N_{s}$ in $s$ 'neighborhood: $\quad i^{t+1}=w_{d} * i_{\text {age }}^{t} *\left(p-i^{t}\right)$, with $w_{d}=0.1 * e^{-\frac{d^{2}}{2 * 0.42^{2}}}, d=$ distance in steps between $s$ and $i$

vi. update edges between $s$ and $i \in N_{s}$ : $\quad$ edge $_{a g e_{s, i}}=$ edge $_{\text {age } e_{s, i}}+1$

vii. update $s_{\text {age }}$ and $i_{\text {age }}, i \in N_{s}$

viii. delete edges with $e d g e_{a g e}>e d g e_{T}$

ix. delete nodes with no edges

(c) remove nodes which have never been selected as best-matching $s$ in (b)

(d) evaluate new accuracy for $M$ as the mean average distance between each node $p \in P_{i}$ and the correspondent best matching unit $s \in M$

while (old_accuracy - new_accuracy $\geq a c c_{T}$ )

3. evaluate the covariance matrix for each cluster (node of the network) by using the associated points from the cloud

4. repeat point 2. using the Mahalanobis distance instead of the Euclidian one in (b).i

The following thresholds and formulae have been used: $a_{T}=0.1$, edge $_{T}=50$, $a c c_{T}=0.001$, age $_{T}=1-\frac{1}{\alpha} *\left(1-e^{\frac{-\alpha * 5}{\beta}}\right), \alpha=1.05, \beta_{w}=3.33, \beta_{n}=14.3$. As discussed in [11], the accuracy threshold $a_{T}$ affects the final model, allowing for multi-scale analysis (although this aspect has not been investigated in this work). The growing steps (b).i-(b).ix follow the algorithm in [11], while points 3) and 4) are introduced to better represent the surface in points of high curvature, as also suggested in [2]. The model can be seen as a classifier, obtained by means of clustering techniques: the next paragraph shows how to adapt it to the other shapes of the training set $T$ (only nodes of the final model are involved in the adaptation phase).

\subsection{Point Correspondence: Adaptation Phase}

In order to perform shape analysis, we need to adapt the model to the other clouds of points, keeping point correspondence. Adapting the network to a new pattern is equivalent to using the model as a classifier: for each given point in the new object, we find the best-matching cluster and adapt it. The process has similarity with SOMs, since there are no added/removed nodes. The segmented patterns $D_{i}$ are considered already normalized for scaling, while we adjust for position and orientation during the process. The main parts of algorithm are summarized:

1. perform block 2 of the algorithm presented in paragraph 2.1, without adding/ removing nodes (point correspondence granted, points 2.b(vii),2.b(ix),2.c skipped)

2. adapt the nodes which were never selected as best matching in the previous step (1)

(a) move each node towards the closest node which was selected in (1)

(b) average the node positions

3. repeat step (1), but using the points which were selected in (1) as new input (instead of the original cloud of points), and adapting only those nodes which were never selected as best matching (nodes involved in step (2))

Steps (2) and (3) are introduced in order to deal with non-closed objects. Performing the adaptation for all the clouds of the training set leads to $n$ neural networks (models) $M_{1}, \ldots, M_{n}$. Summarizing the whole algorithm: while working on the first shape, the 
unsupervised clustering identifies the best location for nodes in order to optimize the representation of the cloud of points. During the adaptation phase, the nodes are moved towards those areas in the new shape where clusters are needed in order to better represent the new cloud of points. Since no nodes are added nor removed, by simply labeling the nodes on the first model and following them during the adaptation we obtain the desired correspondence.

\section{Results}

Results on synthetic datasets. The datasets created for the synthetic experiments are shown in figure $1 \mathrm{a}$. The algorithm introduced in 2.1 involves the randomization of the sequence of surface points (step 2.(a)); randomizing the sequence guarantees a more homogeneous growth for the network, but we have to assure stable results. For each shape, we run the algorithm 20 times using the same sequence of surface points, and evaluated the dissimilarity between models. Table 1 reports the results (stability test): the accuracy for a model, and the dissimilarity between two models $M_{i}, M_{j}$ are defined as:

$$
\begin{array}{r}
\text { accuracy }=\frac{1}{N_{\text {points }}} \sum_{j=1 . . N_{\text {points }}}\left\|p_{j}-w_{j}\right\|, \\
d(i, j)=\text { accuracy }_{i j}+\text { accuracy }_{j i}+\left(1-\frac{E_{m}}{E_{M}}\right),
\end{array}
$$

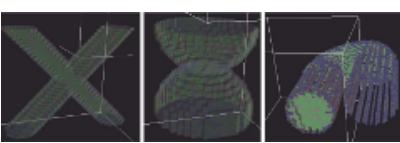

(a)

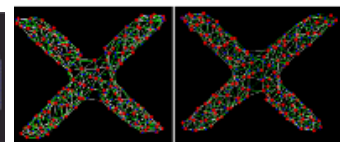

(b)

Fig. 1. (a) Synthetic datasets (dimensions in voxel): from left to right: XShape (radius $=5$, length = 30), Chalice (radius =10), and $U$-tube (radius =10, length =105); (b) Overlapping for best $($ Left , diss. $=2.58)$ and worst $($ Right, diss. $=3.59)$ matching shapes: in both cases, one network has red nodes and white edges, the other has blue nodes and green edges. Even in the worst case the two networks nicely overlap.

Table 1. Results for stability and noise tests (accuracy and dissimilarity are given in voxel); results are averaged over 20 runs per shape and show the stability of the algorithm in relation to

\begin{tabular}{|c|c|c|c|c|c|c|c|c|c|c|c|c|c|c|}
\hline & \multicolumn{9}{|c|}{ Stability Test } & \multicolumn{5}{|c|}{ Small Noise Test } \\
\hline & \multicolumn{3}{|c|}{\begin{tabular}{|l|l|} 
Surf. & Avg. Diss. \\
\end{tabular}} & \multicolumn{2}{|c|}{ Accur. } & \multicolumn{2}{|c|}{$\mathrm{N}$ nodes } & \multicolumn{2}{|c|}{$\mathrm{N}$ edges } & \multirow{2}{*}{\begin{tabular}{|c|} 
Surf. \\
Points
\end{tabular}} & \multicolumn{2}{|c|}{ Avg. Diss. } & \multicolumn{2}{|c|}{ Accur. } \\
\hline & Points & $\mu$ & $\sigma$ & $\mu$ & $\sigma$ & $\mu$ & $\sigma$ & $\mu$ & $\sigma$ & & $\mu$ & $\sigma$ & $\mu$ & $\sigma$ \\
\hline $\mathrm{X}$ shape & 1464 & 2.84 & 0.68 & 2.18 & 0.005 & 146 & 0 & 372.95 & \begin{tabular}{|l|}
7.72 \\
\end{tabular} & 1317 & 3.09 & 0.72 & 2.32 & 0.01 \\
\hline Chalice & 1208 & 3.53 & 0.94 & 2.25 & 0.01 & 120 & $\overline{0}$ & 309.65 & 4.86 & 1087 & 3.04 & $\overline{0.71}$ & 2.39 & 0.03 \\
\hline U-Tube & 2247 & 3.33 & 0.83 & 2.37 & 0.007 & 223.85 & 0.49 & 485.25 & 10.78 & 2022 & 3.12 & $\overline{0.74}$ & 2.53 & 0.03 \\
\hline
\end{tabular}
random effects and noise. Details on the applied randomization and noise are given in the text. 
where $N_{\text {points }}$ is the number of surface points, and $w_{j}$ is the best matching node in the model, given the $p_{j}$ point of the surface sequence (the Euclidian distance is used). In $d(i, j)$, accuracy ${ }_{i j}$ is the accuracy of the model $M_{i}$ evaluated on the set of nodes of the model $M_{j}$ (see eq. 1), $E_{m}=\min \left(E_{i}, E_{j}\right), E_{M}=\max \left(E_{i}, E_{j}\right)$, and $E_{k}=\sum_{q=1 . . N_{\text {edges }}^{k}}\left\|e d g e_{q}\right\|^{2}, k=i, j$. Figure 1 b shows a visual example for the dissimilarity between models. Tests on robustness to noise were performed: for each shape, starting with the original set of surface points, we built up 20 sequences by applying the following noise: $60 \%$ of the points were randomly moved in space (displacement in the range $[-2,2]$ voxels for all the coordinates), and $10 \%$ of the nodes were removed. Table 1 (small noise test) shows the results. Finally, we tested the ability of the graph to adapt to different instances of a same class of shapes (capturing the main variations). We created a dataset of 40 tubes, where each tube varies in diameter and length following Gaussian distributions (radius $r: \mu_{r}=10, \sigma_{r}=2$ voxel, length $l: \mu_{l}=125, \sigma_{r}=40$ voxel). We generated an average tube and used it to train a growing neural network (first model); the model has then been adapted to the 40 tubes (average accuracy for the adaptation process: $\mu_{a c c}=2.37, \sigma_{a c c}=0.2$ voxel). We finally performed a PCA to analyze the performances of the PDM obtained with our algorithm. The properties we tested are the same presented in [6]. Compactness $C(M)=\sum_{m=1}^{M} p^{m}$ (percentage of variance covered by the first M modes); reconstruction error $E_{r}(M)=\frac{1}{N_{s}} \sum_{i=1}^{N_{s}}\left|S_{i}^{\prime}(M)-S_{i}\right|$ (average accuracy in approximating $N_{s}$ shapes of the training set using $\mathrm{M}$ modes); generalization ability $G(M)=\frac{1}{N_{s}} \sum_{i=1}^{N_{s}}\left|S_{i}^{\prime}(M)-S_{i}\right|$ : for a given $i$ we build up a shape model without using $S_{i}$, and test the accuracy with which $S_{i}$ is approximated by the model $\left(S_{i}^{\prime}(M)\right)$; specificity $S p(M)=\frac{1}{N} \sum_{i=1}^{N}\left[\operatorname{Diss}\left(S_{i}(M)\right.\right.$, ObjCls $\left.)\right]$, with
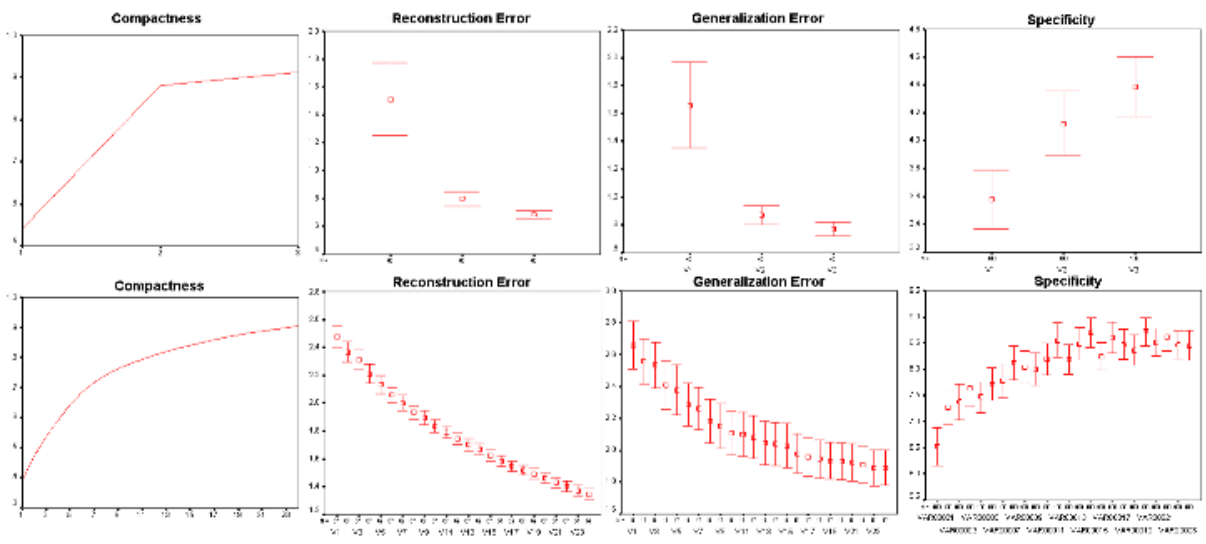

Fig. 2. First row: quantitative analysis for the Tube-PCA: compactness (in percentage), reconstruction error, generalization ability, and specificity (in voxel) are reported. The $x$ axis shows the retained modes of variation. The error bar shows the confidential interval at $95 \%$ (see text for details). The model identifies the 2 main modes of variation. Second row: same analysis for brain ventricles (values are in $\mathrm{mm}$ ): when the 24 modes of variation are used, the error values are particularly low, showing the good behaviour of the model: $E_{r}<1.4, G_{e}<2$, and $S p<9$. 


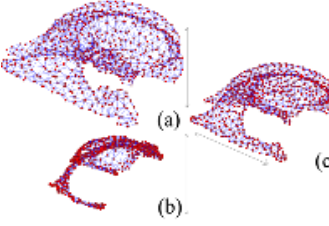

I

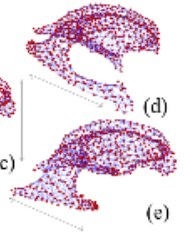

(e)

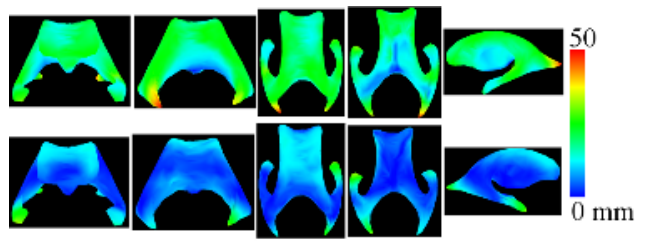

II

Fig. 3. Left image I: (a)-(b) $1^{\text {st }}$ mode of variation, $\pm 3 \sigma$; (c) mean shape; (d)-(e) $2^{\text {nd }}$ mode of variation, $\pm 3 \sigma$. The $1^{\text {st }}$ mode presents a global shrinking/growing behaviour, while the $2^{\text {nd }}$ mode shows elongation/compression of the temporal and occipital horns; Right image II: Color-coded maps for the amount of movement of each node. Top row: $1^{s t}$ mode $\pm 3 \sigma$. Bottom row: $2^{n d}$ mode $\pm 3 \sigma$ (occipital and temporal horns mostly involved).

$\operatorname{Diss}\left(S_{i}(M), O b j C l s\right)=\frac{1}{N_{s}} \sum_{i=1}^{N_{s}}\left|S_{i}^{\prime}(M)-S_{i}\right|$, where we create $N=100$ instances of the object class and average the dissimilarity between them and the shapes in the training set. The results are shown in figure 2

Results on a medical dataset. We applied the algorithm on a training set of 67 , semiautomatically segmented, brain ventricles (T2-MRI, 91×109x91 voxels at $2 \times 2 \times 2 \mathrm{~mm}$ after normalization). After acquiring the clouds of points for each ventricle, we selected a representative one and build up a first model (see section 2.1): the optimal number of clusters (nodes) resulted in 755. The model was adapted to the other 66 clouds, and a PCA 1 was performed on the 67 models (average accuracy for the adaptation resulted in $\mu_{a c c}=2.06, \sigma_{a c c}=0.22 \mathrm{~mm}$ ). Results are reported in figure 2, while figure 3 shows the first 2 modes of variation. Although it was not the aim of the study, we tested the linear correlation between aging and first mode of variation. The dilation was significantly associated with aging ( $p<0.01, r^{2}=0.10$, older people showing a larger structure). The low value for $r^{2}$ is explained by the low variation in the population $(\mu=75, \sigma=5$ years).

\section{Discussion}

In Kaus et al. [2], the authors iteratively reduce the set of original surface points to a subset with high (sparse) dense point distribution in areas of high (low) curvature: a triangular mesh is then derived. In our method, all the surface points are used, and the unsupervised clustering detects the areas in which more clusters (nodes) are needed. Differently from [2], we adapt nodes as in SOM: once a node is moved, a parameter controls how many neighbors have to move, and a Gaussian function is used to determine the strength of movements. As in [2], we also make use of an elastically deformable model which avoids inverse transformations. In Gerig et al. [12], spherical harmonics are used to model a set of surface points. The main advantage is the multiscale analysis which might highlight deformations not noticeable otherwise; drawback

\footnotetext{
${ }^{1}$ While applying the PCA, we adjusted for translation and rotation of shapes following the procedure suggested by Evangelos et al. in [13].
} 
of the solution are pre-processing steps needed for closing the surface. We have not investigated the multi-scale feature, although we speculate it would be achievable by a reduction in the accuracy for the growing process $\left(a_{T}\right)$. Our solution does not require a closed surface. Finally, the parameters used in our approach are the coordinates of the nodes: significant differences in shape are directly associated with positions, (in [12] a vision inspection is needed). In [8], Davies et al. highlight how critical some aspects are for the final model: the number of nodes, their locations, and the correspondence between them strongly influence the final performances. The authors suggest to use $d e$ scriptive functions to identify correspondent points on different shapes, create a first model, and evaluate it through an objective function to be optimized: the search for the best descriptive functions is carried on through genetic algorithms. This approach leads to the distinction between pairwise and groupwise solutions. Groupwise approaches are known to provide better models, optimizing the results on the whole dataset; moreover, they do not require a first representative to start with. Pairwise approaches, like the one described in this work, need an initial shape to operate: thus, the final model can be influenced by such a choice. Nevertheless, pairwise approaches might overcome some drawbacks related with groupwise solutions. The optimization needed by groupwise solutions is often achieved by genetic algorithms (see [8]): although this improves the chances of getting a better model, an optimal result is not guaranteed. Genetic algorithms lead to heavy computation, surely a drawback if we aim to learning systems: how can we improve a given model when a new example becomes available? Groupwise solutions have to undertake the whole optimization process again, with a computation load which grows with the number of shapes. Pairwise and groupwise solutions should be seen as complementary for many aspects, and the results achieved in one field can improve the other. The results reported in sections 3 show the stability of our solution and its capability of generating good point distribution models (PDM) for statistical analysis (such as PCA). The stability has been tested on synthetic datasets both with and without noise, while the PDM has been tested both on synthetic dataset and on a medical training set of brain ventricles. The PCA could highlight the expected modes of variation for the synthetic data set and known variations in the aging brain ventricles due to brain atrophy.

\section{Conclusions}

In this paper, we presented a new solution, rooted in a pattern recognition framework, for a pairwise approach to the shape-modelling problem. An unsupervised clustering technique (SONGWR) automatically identifies the optimal number of nodes and their locations on the surface of a representative shape; the point distribution model (PDM), considered as a self-organized map (SOM), is then adaptated onto other datasets: the correspondence problem is solved by labeling the nodes in the first model and following them through the adaptation phase. The method has been thoroughly evaluated on synthetic datasets and its effectiveness has been proved in a challenging medical application. Due to its generality, the method can be applied to other anatomical structures (of any shape). Further investigation will be focused on the integration of the image intensities around the nodes in the model. 


\section{Acknowledgment}

This work was supported by the Technology Foundation STW, applied science division of NWO and the technology programme of the Ministry of Economic Affairs, and by Medis medical imaging systems, Leiden, The Netherlands (www.medis.nl). The authors would like to thank W.M. Palm, M.D. for providing the medical dataset and for his help during the segmentation process.

\section{References}

1. T. F. Cootes, C. J. Taylor, D. H. Cooper, J. Graham, Active Shape Models - their training and application, Computer Vision and Image Understanding, Vol. 61, no. 1, January, pp. 38-39, 1995

2. M.R. Kaus, V. Pekar, C. Lorenz, R. Truyen, S. Lobregt, J. Richolt, J. Weese, Automated 3D PDM Construction Using Deformable Models, proceedings of the Eigth IEEE International Conference on Computer Vision (ICCV'01) pp. 566-572 vol.1

3. A. Pitiot, H. Delingette, A. W. Toga, P.M. Thompson, Learning Object Correspondences with the Observed Transport Shape Measure, IPMI 2003, pp. 25-37

4. S. Wrz, K. Rohr, Localization of Anatomical Point Landmarks in 3D Medical Images by Fitting 3D Parametric Intensity Models, IPMI 2003, pp. 76-88

5. D. Rueckert, A. F. Frangi, J. A. Schnabel, Automatic Construction of 3-D Statistical Deformation Models of the Brain Using Nonrigid Resitration, IEEE Transactions on Medical Imaging, vol. 22, no. 8, August 2003, pp. 1014-1025

6. R.H. Davies, C. J. Twining, P. D. Allen, T. F. Cootes, C. J. Taylor, Shape Discrimination in the Hippocampus Using an MDL Model, IPMI 2003, pp. 38-50

7. H. H. Thodberg, Minimum Description Length Shape and Apperance Models, IPMI 2003, pp. 51-62

8. R. H. Davies, C. J. Twining, T. F. Cootes, J. C. Waterton, C. J. Tayolr, A minimum description length approach to statistical shape modelling, IEEE Transactions on Medical Imaging Vol.21, pp.525-537, 2002

9. B. Fritzke, Growing Cell Structures - A self-organizing Network for Unsupervised and Supervised Learning, Neural Networks (1994), Vol. 7, No. 9, pp 1441-1460

10. B. Fritzke, Kohonen Feature Maps and Growing Cell Structures - a Performance Comaprison, NIPS 1992, Denver.

11. S. Marsland, J. Shapiro, U. Nehmzow, A self-organizing network that grows when required, Neural Networks 15 (2002), pp. 1041-1058

12. G. Gerig, M. Styner, D. Jones, D. Weinberger, J. Lieberman, Shape analysis of brain ventricles using SPHARM, Published MMBIA, IEEE Computer Society: 171-178, Dec. 2001

13. E. A. Coutsias, C. Seok, K. A. Dill, Using Quaternions to Calculate RMSD, Journal of Computational Chemistry, 2004, vol 25, pp. 1849-1857 